\title{
Lift the Grit's Veil: The Literature Review and Prospects of Grit
}

\author{
Qiurong Liang \\ School of Management, Jinan University, Guangzhou, China \\ Email: liangqiurongjnu@foxmail.com
}

How to cite this paper: Liang, Q. R (2021). Lift the Grit's Veil: The Literature Review and Prospects of Grit. Psychology, 12, 580-594.

https://doi.org/10.4236/psych.2021.124036

Received: February 24, 2021

Accepted: April 20, 2021

Published: April 23, 2021

Copyright $\odot 2021$ by author(s) and Scientific Research Publishing Inc. This work is licensed under the Creative Commons Attribution International License (CC BY 4.0).

http://creativecommons.org/licenses/by/4.0/ (c) (i) Open Access

\begin{abstract}
In the era of the Internet, telecommuting, virtual office and other work modes are increasingly popular. Telecommuting and other working modes give employees more freedom and flexibility in work, which reduces the direct monitoring from the outside. The complex network information will also bring interference and task interruption. At this time, how to maintain the focus on the task and self-motivation becomes particularly important. Grit refers to the perseverance and passion for long-term goals. As a variable of self-driven orientation and future perspective, it is a psychological resource to boost the realization of goals. Firstly this paper analyzes the concept, variable differentiation and measurement of grit. Secondly, the positive influence of grit on the realization of goals is discussed. Finally, this paper discusses the practical significance and future research prospects.
\end{abstract}

\section{Keywords}

Grit, Passion, Goal, Control Mechanism, Attention Mechanism

\section{Introduction}

In the current information age of the interconnection of everything, the massive information environment of the Internet presents the characteristics of dynamic, complex, uncertain and ambiguous, which increases the information interference and temptation in the work for employees. At the same time, the growing popularity of work patterns, such as online office, has allowed workers to work more flexibly on working hours and workplaces, with less direct supervision from their leaders. Under such a working background, it is particularly important for individuals to keep persevered and stick to their original goal, so as to insist on achieving the goal. In our daily lives, we all typically have a number of goals we want to achieve. For example, performing well in sports, achieving 
work targets, and maintaining good health are, for many people, an indispensable part of daily life. Despite having great importance, people often abandon their already set goals. Therefore keeping perseverance and passion for Long-Term Goals has become especially valuable in today's society. Duckworth et al. (2007) put forward the concept of grit, which refers to perseverance and passion for long-term goals. Research shows that grit has shown effective and positive effects on organization members in many fields such as medical treatment, education and enterprise. In the medical field, nursing workers with high levels of grit are believed to make better use of their abilities and be less frustrated by common failures and setbacks, resulting in greater personal achievement and lower job burnout (Seguin, 2019). In the field of education management, novice teachers with high scores of grit have higher work efficiency and retention rate (Duckworth et al., 2009). While in the field of enterprise management, scholars have found that employees with higher levels of grit have higher job engagement and job performance (Suzuki et al., 2015), low likelihood of changing careers or jobs (Burkhart et al., 2014), more job stability (Duckworth et al., 2007), less counterproductive behavior (Andrea et al., 2016), and good occupational health (Kim \& Lee, 2015).

Since Duckworth et al. (2007) propose the concept of grit, it began to increasingly become one of the hot issues in the western management research, despite there are many empirical studies of grit, but there is no research that reveals the black box of grit how to work, and study the importance of girt on organization under the era of digital economy. Grit is closely related to goal achievement, emphasizing persistence and passion for long-term goals. To be specific, Gritty employees can control, regulate and manage themselves around work goals, which is a kind of self-goal-oriented psychological resource. Gritty employees are often shown to have outstanding management and control ability for work tasks and objectives. Generally speaking, these employees will take the initiative to set reasonable work goals, and will consciously supervise and complete the goals. Even in the face of difficulties, they will persist and take the initiative to find effective solutions, which undoubtedly is of positive significance for employees to complete their work tasks. Given the close link between grit and goal, this article based on the literature review, firstly analyzes the concept, variable differentiation, structure and measurement of grit; secondly, the positive influence of grit on goals is discussed from the perspectives of goal self-correction and goal self-promotion, and the mechanism behind it is summarized. Finally, this paper discusses the practical significance and future research prospects, in order to provide reference for promoting the effective management of human resources in the environment of mass information on the Internet.

\section{The Concept and Measurement of Grit}

\subsection{The Concept of Grit}

"Grit" is first put forward by Duckworth et al. (2007), refers to the perseverance 
and interest towards long-term goals. Based on the interviews of a lot of successful celebrity, including business elite, artists, educators, athletes and so on, Duckworth et al. (2007) found that they all have one thing in common, namely, despite the failure, adversity and stagnant, will try to cope with the challenge. The essence of grit is a commitment to a long-term goal. Individuals with high levels of grit are more likely to view the process of achieving goal as a marathon rather than a sprint, perseverance and passion are their strengths. Gritty individuals are more likely to persist and ultimately achieve their goals than those who change course or stop when frustrated or bored.

After the concept of grit was put forward, many scholars conducted relevant studies and distinguished and analyzed its related variables, such as conscientiousness, self-control and so on. First, grit is different from conscientiousness. In a sense, grit and conscientiousness are similar, but their orientation and nature are different. In pursuit of goals, grit people focus more on long-term endurance than short-term strength (Duckworth et al., 2007). Moreover, conscientiousness does not reflect the goal-orientation underlying the concept of grit. Grit is about maintaining an interest over a long period of time, even when there is difficulty or no positive feedback. In fact, high levels of grit were shown by outstanding people in every field (Duckworth et al., 2007). Whether or not extrinsic rewards are lacking, they can do hard work in their field for a long time and keep passion. While consciousness, as part of the Big Five, is a personality trait. Conscientiousness may involve many aspects of short-term trivial work, emphasizing that the individual's behavior complies with the norms prescribed by society (Morton, 2014). However, grit emphasizes more on goal orientation, focusing on the long-term adherence to the goal rather than the short-term (Duckworth et al., 2007). In conclusion, conscientiousness does not reflect the underlying concepts of grit, such as goals and passion (Fite et al., 2017). Secondly, there is a difference between grit and self-control (Duckworth et al., 2007). Previous studies have shown that grit is closely related to self-control (Duckworth, 2016). People with high levels of self-control may have the ability to regulate attention and resist temptation, but that doesn't necessarily mean they're grit when it comes to achieving a particular goal in life. Therefore, the correlation between grit and self-control is different in terms of action and commitment (Duckworth, 2016). In terms of emotion, self-control does not involve passion. Some people have high self-control but lack the passion of goal and may live a life without passion (Duckworth, 2016). In terms of time, self-control means ignoring the temptations of the moment. This control may be more short-term in terms of time, whereas grit involves pursuing higher-level goals over a longer time. In terms of regulating orientation, self-control focuses on supervising oneself not to do bad things, while grit not only emphasizes not to do negative things, but also emphasizes to actively seek and take positive behaviors (Vainio, \& Daukantaite, 2015). Moreover, grit is different from growth mindset. Generally speaking, people who hold a growth mindset believe that abilities are prop- 
erties that can be increased, molded and manipulated. In other words, an individual's ability can be constantly improved through hard study and training. They see difficulties and failures as challenges that help them grow, so they may choose more challenging and creative work. Grit is associated with growth thinking and growth mindset can promote grit (Duckworth, 2016). They focus on their own development and are accustomed to comparing themselves with their past rather than relying on external standards, thus presenting a state of progress. Besides, they are able to find effective strategies in the face of situational pressures and downturns. In other words, people with growth mindsets can overcome obstacles and are less likely to give up in frustration, which enables to stick to a long-term goal. To sum up, even if related to other variables, grit is a different concept. A kind of unique positive psychological variable is defined as persistence and passion for long-term goals.

\subsection{The Measurement of Grit}

The self-report method is mainly used for the measurement of grit, among which the scale method is the most widely used, and the grit scale developed by Duckworth is more representative. Initially, the author wanted to find a scale to measure grit that could meet the following four criteria: 1) applicable to different fields, whether school or business; 2) suitable for different people; 3) accurately fit the two dimensions of grit; 4) effectively distinguish other variables. Based on the above considerations, Duckworth et al. (2007) developed the original scale of grit (Grit-O) on the basis of multiple data collection and analysis, including two dimensions of perseverance and passion. There are 6 items for each dimension. The perseverance dimension entries such as "I can accomplish goals that will take years". While passion dimension includes items, such as "I was fascinated with an idea or project for a short time, but then lost interest", the scale is scored on a five-point Likert scale, with 1 being very unlike me and 5 being very like me. The higher the score, the higher the level of grit.

With the further development of the research, Duckworth, Quinn and Seligman (2009) developed a more effective Grit Short Version (Grit-S) on the basis of the original scale, which retained the two-factor structure of the original scale, eliminated 4 items and retained 8 items. The Grit-S scale fitted the data better than the Grit-O scale, and the reduction of items did not reduce its predictive validity. Due to its superior psychometric characteristics、 better predictive validity and fewer items, this scale has been adopted by many researchers, and empirical studies show that this scale has high reliability. On the basis of previous scales, later scholars further refined the scale and revised the version of the scale to adapt to different cultural backgrounds according to the research needs. They include German (Fleckenstein et al., 2014), Korean (Kim \& Lee, 2015), Japanese (Kazuji, Shiori, \& Toshihiko, 2015), Turkish, Russian (Tyumeneva et al., 2014) and Spanish (Karaman et al., 2018). Therefore, the grit scale has been used in different cultural backgrounds. 


\section{The Positive Effects of Grit}

As mentioned above, previous studies have shown that grit has positive effects in a number of areas, including education, the workplace, and health care. In the field of education, grit is an important determinant of academic success and achievement, such as academic productivity and engagement (Hodge, et al., 2018); Learning motivation (Eskreis et al., 2014); Academic performance; Time devoted to learning; Strategies for learning (Weisskirch \& Robert, 2016); in the medical industry, grit also plays a positive role. Some research suggests that grit, as a form of resilience, can reduce suicidal ideation in response to negative life events. Kleiman, Liu, and Riskind (2014) used a longitudinal study to explore the relationship between grit, gratitude and suicidal ideation, and found that subjects with high grit scores had lower suicidal ideation. This suggests that grit may be a useful diagnostic predictor or buffer against suicidal ideation. Grit people are less affected by negative life events for several reasons. First, grit people are more likely to persist in the face of setbacks and adversity. Although, like everyone else, gritty people also find negative events painful, they may be more likely to persist or change their direction rather than abandon goal-related efforts. Previous research has shown that this kind of personal initiative and flexibility can prevent suicide when faced with goal barriers. If grit brings perseverance and hope, it may be a protective factor against suicide. Secondly, grit and gratitude allow individuals to create and maintain life goals by giving them a greater sense of meaning in life. These goals are the primary source of meaning and purpose in life. Grit people have meaningful pursuits that enable them to bridge the gap between who they are and who they want to be. Third, grit people are less affected by negative events. They may minimize their focus on stress by focusing on a longer term goal. In addition, grit may be imbued with resilience through internal motivation and Interpersonal interaction. Grit people may be more active in reframing negative events, or be able to solve problems effectively even in high-stress situations. For example, by highlighting long-term goals, grit can motivate individuals to overcome obstacles to achieving their long-term goals. Emphasizing the importance of long-term goals can motivate individuals to build a social support system or other beneficial resources before the goals are achieved. These resources can be used in the event of a negative life event. Studies have shown that people with grit are better able to endure unpleasant experiences, and this may explain why people with grit have a higher success rate, thus confirming the potentially beneficial effect of grit (Duckworth, 2016). In the field of workplace, studies have explored the relationship between grit and job satisfaction, job achievement, work burnout, job engagement, job performance (Suzuki et al., 2015; Andrea et al., 2016); In addition, grit has a positive psychological effect, which is reflected in cognitive and emotional aspects. For example, grit can enhance Mental health (Sharkey et al., 2017); Self-efficacy (Muenks et al., 2017); Self-esteem (Weisskirch \& Robert, 2016); Growth mindset. 
Overall, gritty employees are more able to resist and correct their own deviation activities in a timely manner on the one hand; on the other hand, they are more able to actively think and initiate their own goal promotion activities, so that it is easier to achieve their goals. That is to say, gritty employees have obvious self-resistance and self-promotion effects on goals.

\subsection{Correction Effect of Grit on Goal Deviation}

First of all, grit emphasizes the overcoming of negative cognition and negative behavior that deviate from the goal. When faced with setbacks at work, gritty employees have stronger ability to resist negative influences. This strong ability is reflected in: gritty employees are less likely to have negative cognition and negative behavior due to setbacks at work, and gritty employees have stronger ability to adjust themselves after setbacks. First, gritty employees are less likely to quit due to job setbacks. Due to the persistence of the goal, the gritty individuals tend to actively seek resources to solve the problem when they suffer setbacks, rather than choose to escape. Therefore, in the workplace, gritty individuals have lower demission intention and low turnover rate (Burkhart et al., 2014; Salles et al., 2014, 2016; Jeong et al., 2019). A survey of 288 hotel managers shows that managers with higher levels of grit are more insistent in times of insecurity and are less likely to make personnel changes and career changes. In addition, gritty employees experienced less burnout or were better able to resist the negative effects of burnout. In the medical industry, some scholars adopted a cross-sectional research design and took 548 doctors as subjects to discuss the relationship between doctors' grit and job burnout (Halliday et al., 2017). The results show that grit is negatively correlated with job burnout, and individuals with grit are less likely to feel job burnout. Scholars on the basis of further explore the influence of fortitude and counterproductive behavior, for example, Andrea et al. (2016) make a survey of 208 private service industry employees, in the relationship between job burnout and counterproductive behavior, even if the same in the case of job burnout, compared with the low level of grit, people with higher levels of better control and adjustment, appear less counterproductive behavior (Andrea et al., 2016).

Employees with higher levels of grit are better able to recover from negative emotions. Gritty individuals always pursue long-term goals in their hearts, so they will have a sense of immersion in this process and will not be easily troubled by the outside world, especially things unrelated to the goals. Studies have shown that gritty individuals have higher emotional stability in the face of stressful or negative events. Stoffel and Cain (2018) studied the impact of negative performance feedback on follow-up performance tasks and the moderating effect of persistence. the research found that when the grit level is high, the negative feedback through sadness has little effect on task performance, and when levels of grit is low, negative feedback through sadness bigger effect on task performance. In addition, when the relationship between superiors and subordi- 
nates or colleagues in the workplace is not very good, compared with the employees with low level of grit, the employees with high level of grit are better able to adjust or reduce the impact of the unsatisfactory relationship, maintain consistent enthusiasm and maintain a high level of work engagement. Negative emotions will distract attention, occupy attention resources, lead to withdrawal behavior, and reduce the durability of the task, while grit people have the ability to focus on task goals, they are less likely to let sadness interfere with their attention or problem-solving ability, so grit people show a stronger ability to recover from stress or negative emotions (Stoffel \& Cain, 2018).

\subsection{The Promoting Effect of Grit on Goal Approach}

Grit emphasizes the initiation of positive cognition and active behavior toward the goal. First, Gritty leaders exhibit more active leadership and initiative. Schimschal and Lomas (2019) conducted a study with 100 leaders from different industries. The subjects completed the grit Scale and Positive Leadership Self-Rating Scale. The results show that grit is positively correlated with positive leadership, and gritty leaders have stronger positive leadership ability. Grit has a stronger correlation with positive meaning, that is, leaders can gain a sense of meaning from helping others succeed and reflecting on their own path to success, thus maintaining enthusiasm for their goals. Seguin (2019) study the nurse's direct leader, the results showed that the nurse leader' grit score on a leadership role with its increased with the extension of working life and the increase of personal accomplishment, and moreover, gritty nurse leader is more likely a lifelong learner, in their career to pursue a higher degree.

Second, gritty employees show more job involvement and organizational commitment. According to the research of Seo et al. (2014), grit will strengthen the organizational commitment of employees. Employees with high levels of grit are more serious in their work, more involved in the workplace and have much higher commitment and loyalty to the organization than employees with low grit. Employees with high levels of grit show more positive and constructive work behaviors, such as voice behavior. Grit is a combination of perseverance and passion for long-term goals. and though they may face challenges and resistance, is likely to take risks for their employees and organizational improvement Suggestions, and it is through organizational commitment play a role in this process, if the employee is very loyal to an organization, they will feel improve my advice is to make more contribution to the development of the organization's goals (Andrea et al., 2016). In short, in the process of goal promotion, gritty individuals are more far-sighted and possess a higher level of growth thinking (Duckworth et al., 2007), show a higher level of commitment to the organization, more proactive work thinking, higher job satisfaction, and better job task performance (Suzuki et al., 2015; Jachimowicz et al., 2018; Holdan et al., 2018). 


\section{The Mechanism of Positive Effect of Grit}

Duckworth et al. (2007) believe that grit has two dimensions, namely: the consistency of passion, which reflects the individual's tendency to keep commitment and focus on achieving goals/tasks over a long period of time. Persistence in effort reflects a person's tendency to pursue long-term goals through sustained efforts despite obstacles and setbacks. Corresponding to the two dimensions of grit, this paper holds that the mechanism of grit includes two aspects: the attention mechanism of goal targeting and the control mechanism of goal maintaining.

Goal is an important concept in management and psychology research. It refers to the thing or state that people strive to pursue and can guide their current behavior. It mainly solves the problem of "why do people act". Scholars have emphasized the value of goals for individuals in different studies. For example, psychologist Adler once said, "If a person has no goals, he cannot think, feel, hope or act". By connecting individual's will with action, goal endows meaning to individual's action and even life, and therefore becomes an important perspective to understand individual behavior. At the same time, goal theory has become an important theoretical basis for understanding human motivation and personality, and has been applied more and more widely in the study of organizational behavior, and has increasingly become an important theory with fundamental significance. Goal-driven results are behind people's certain motivations or behaviors, so is the formation of positive role of grit which is also goal-driven results. Therefore, we believe that the goal theory is an ideal theoretical basis and theoretical perspective to explore the formation mechanism of the positive after-effect of grit.

\subsection{Goal Targeting Mechanism: Consistency of Interest}

Consistency of interest is an important dimension of grit. Duckworth and Eskreis-Winkler (2015) suggest that grit individuals give up something they don't love, but that giving up may help them better stick to what they really care about, whether it's a person's career or their schooling. Gritty employees are committed to work they love, believe they can achieve their goals, and are willing to change their strategy or behavior to achieve those goals. In other words, gritty individuals tend to have a mature capacity for action, and they are able to change lowand medium-level goals because they have a sense of agency and planning that incorporates future thoughts and actions. In a sense, gritty individuals have a higher level of goal targeting, they love that goal and they are committed to achieving it. Due to the consistency of interest, the members of the firm focus more on the long-term goals, which is the goal targeting mechanism of grit.

\subsection{Goal Maintenance Mechanism: Perseverance of Effort}

Perseverance of effort is another important dimension of grit. gritty employees can work in adversity to achieve higher goals (Sheldon et al., 2015), and to better cope with stress (Sharkey et al., 2017), namely the consistency level higher em- 
ployee encountered trouble in work, they are more likely to seek out different strategies to cope with the situation, in a less stressful way to adapt and spent. We believe that persistence in the face of work adversity embodies a strong goal maintenance mechanism. Specifically, grit employees reinforce their commitment to work goals in at least three ways. First, they are more likely to ignore negative events. Grit employees may be able to focus on longer term goals, thus focusing less on stress and being less distracted by negative and irrelevant events. Secondly, they have a stronger tendency to actively reconstruct negative events. They may reconstruct negative feedback more actively, or solve problems more effectively when the tension caused by negative feedback is high, so they are more able to reduce and restore negative emotions, and have more psychological energy. Thirdly, they have a stronger tendency to actively seek resources. In the process of pursuing goals, they are more focused on the goals and have a higher degree of investment. They are prone to produce more positive emotions, and these positive emotions will further construct more positive psychological resources, thus achieving a virtuous cycle. For example, after receiving negative feedback, gritty individuals may be particularly active in seeking out things that mitigate the negative impact, such as social support (Kleiman et al., 2014). Therefore, due to the perseverance of effort, gritty employees are more determined to work hard so as to achieve long-term goals, which is the goal maintenance mechanism of grit.

\subsection{Interaction between the Two Mechanisms}

Goal targeting mechanisms and goal maintenance mechanisms are not isolated from each other, but mutually reinforcing. On the one hand, the goal targeting mechanism of grit to long-term goals promotes goal maintenance by facilitating the reconstruction of negative feedback. Since grit focuses attention on long-term goals, even when focusing on immediate negative feedback, it means that people with grit focus and care more about long-term goals and are not frustrated by short-term goals. By keeping long-term goals salient, grit can motivate individuals to address problems that impede progress on long-term goals. Such future positioning and utilization of resources has been reflected in other areas of life, such as scholars show long-term academic persistence in the face of adversity. By contrast, people who lack grit may neglect important long-term goals in favor of short-term ones, such as avoiding the consequences of some negative feedback. On the other hand, the maintenance mechanism of grit for long-term goals promotes the goal-targeting mechanism. Persevered employees' continuous efforts toward long-term goals will increase employees' workplace immersion, increase job engagement, and further focus on long-term goals.

\section{Practical Implications of How to Promote Pattern of Grit}

Existing studies have shown that the level of employee grit is not unchangeable, but can be cultivated (Duckworth et al., 2007). Therefore, through necessary 
management design, organizations can promote and cultivate employees' grit at individual level, team level and enterprise level.

\subsection{On the Personal Level: Deliberate Practice}

Duckworth (2016) points out that grit can be increased through repetition and deliberate practice. Grit can change with age and experience. Moreover, some scholars believe that due to the existence of feedback mechanism in the workplace, the stability of grit may change with the passage of time (Crede et al., 2017). Policymakers have also focused on developing programmes to improve the grit of children and adults. Therefore, at the individual level, you can increase grit level by deliberate practice, and then achieve your goals better.

\subsection{Team Level: Learning Goal Orientation}

Existing research distinguishes the relationship between learning goal orientation, which aims to achieve the goal of ability and acquire knowledge and grit, and points out that learning goal orientation may be better to promote grit. Learning goal orientation can help individuals more actively deal with negative feedback (Latham \& Pinder, 2005), and thus cultivate grit. Therefore, from the team level, establishing a learning goal-oriented atmosphere and building a learning goal-oriented team can help activate the grit of employees and cultivate their growth mindset.

\subsection{Corporate Level: Positive Leadership}

In an increasingly globalized world, leaders are increasingly required to work consistently at a high level of grit. Leaders need to demonstrate positive leadership that embodies both enthusiasm and persistence. Positive leadership aims to emphasize the positive orientation in the organization and is committed to demonstrating a positive atmosphere, relationship, communication and vision. On the other hand, grit emphasizes future-oriented, can trigger people to generate self-drive and achieve goals through self-management, which coincides with positive leadership. Recent studies have shown that the level of leadership's positivity is related to the level of employee's grit (Schimschal \& Lomas, 2019). Both perseverance and passion for long-term goals are emphasized. Only to insist there is no passion, could go into a "sadhu" practice. Only the perseverance without passion can lead to the loss of personal life and social creativity, happy is difficult to realize the pursuit of goals and promote self-efficacy, difficult to reach the top goal (Kim, Choi, \& Vandenberghe, 2018). Of course, only passion without perseverance cannot achieve the lofty vision. In view of this, in the process of implementing positive leadership, on the one hand, employees are encouraged to set lofty ambitions, emphasize unremitting efforts. On the one hand, the premise of encouraging employees to persist is to find the entry point of personal love, so as to achieve the effective combination of passion and perseverance. In short, from the perspective of the enterprise, positive leadership 
that emphasizes perseverance and enthusiasm at the same time helps to set a firm example for employees and boost their grit level.

\section{Future Research Prospects}

\subsection{Improve the Measurement of Grit}

Perseverance of effort and consistency of interests are the two wheels in the pursuit of a long-term goal. Some scholars believe that only the combination of the two can fully predict achievement. Some research results are inconsistent because they emphasize too much on perseverance of effort or consistency of interests and fail to be effectively combined to measure (Jachimowicz et al., 2018). Therefore, future research needs to improve the scale of grit, and improve the accurate capture of the two-dimensional connotation of grit. In addition, future research should also focus on the cross-cultural applicability of the positive effect of grit. Culture affects people in all aspects. The "grit" contained in western culture may be different from that in eastern culture. Duckworth (2016) assumed that there were no significant differences between different demographic and socioeconomic status groups. However, evidence suggests that culture-based "goals" and the "goals" described by grit itself may differ significantly from culture to culture. In collectivist cultures, people are more likely to prioritize goals that align with the goals of others. Hence, we encourage future research to look at whether the meaning of goal and grit has the same effect across different cultures.

\subsection{Explore the Dark Side of Grit}

Scant research has explored whether or not grittiness comes with hidden costs, and is linked to undesirable traits. Is there a dark side to grit? What are the inflection point and boundary from positive effect to negative effect? In the current mainstream research, more attention is paid to the positive side of grit, few researchers have discussed the negative consequences of grit. There are increasing calls to reconsider the linear assumptions implicit in organizational science. Scholars also advocate looking at the positive after-effects of grit within the framework of "too much of a good thing" (Pierce \& Aguinis, 2013). Based on this view, grit may trigger negative performance at work under certain conditions. The grit effect may have an optimal advantage, and then decline with changes in mood and environmental dynamics. For example, persistence and stubbornness associated with grit can lead to an individual's unwillingness to "let go and accept failure" (Alaoui \& Fons-Rosen, 2016). In addition, studies have reported a relationship between grit and risk-taking behavior, with grit individuals sticking to goals and being more grit in the face of losses or risky behaviors, which may have negative consequences (Lucas, Gratch, Cheng, \& Marsella, 2015). Because gritty individuals have a complex hierarchy of personal goals and are passionate about them, and may be less receptive to the needs and desires of employers and colleagues, individuals with excessive grit may lack flex- 
ibility and be hampered in transitioning between tasks in different roles. Therefore, future research should try to explore the dark side of grit and the possible overkill effect during different fields.

\subsection{Expand the Research of Grit at Team-Level}

Current studies have mainly focused on the role of grit at the individual level, and future studies may try to explore the role of grit at the team or organizational level. Team level grit may influence group work attitudes and behavior as well as individual level constructs. Teamwork differs from individual work in that it involves the interaction of multiple members, each with their own personality traits, experiences, working styles and varying levels of grit, who must act together to achieve a common team goal. Does consistency of team grit contribute to team performance? When there are differences in the level of individual grit in a team, does it lead to team conflict? What leadership style is the best match for a grit team? These are noteworthy. Some scholars have started to study the influence of start-up team's grit on team performance. Some scholars have also found that entrepreneur's grit influences entrepreneurial success through goal setting and self-efficacy. Nowadays, the development of enterprises and teams is more dynamic and flexible, while grit at the team or organizational level is complex and requires multi-perspective thinking (Wright \& Cropanzano, 2004). Deepening the research on grit at the team level is conducive to exerting the positive effect of grit on work performance and achievement of work goals. Therefore, future research can try to explore the effect of grit on team level from the perspective of interpersonal interaction.

\section{Conflicts of Interest}

The author declares no conflicts of interest regarding the publication of this paper.

\section{References}

Alaoui, L., \& Fons-Rosen, C. (2016). Know When to Fold 'em: The Grit Factor. Working Papers.

Andrea, C., Riccardo, S., Stephan, D., \& Arianna, C. (2016). Grit or Honesty-Humility? New Insights into the Moderating Role of Personality between the Health Impairment Process and Counterproductive Work Behavior. Frontiers in Psychology, 7, Article No. 1799. https://doi.org/10.3389/fpsyg.2016.01799

Burkhart, R. A., Tholey, R. M., Guinto, D., Yeo, C. J., \& Chojnacki, K. A. (2014). Grit: A Marker of Residents at Risk for Attrition? Surgery, 155, 1014-1022. https://doi.org/10.1016/j.surg.2014.01.015

Crede, M., Tynan, M. C., \& Harms, P. D. (2017). Much Ado about Grit: A Meta-Analytic Synthesis of the Grit Literature. Journal of Personality and Social Psychology, 113, 492-511. https://doi.org/10.1037/pspp0000102

Duckworth, A. L. (2016). Grit: The Power of Passion and Perseverance. New York: Simon and Schuster. 
Duckworth, A. L., \& Eskreis-Winkler, L. (2015). Grit. In J. D. Wright (Ed.), International Encyclopedia of the Social and Behavioral Sciences (Vol. 10, pp. 397-401). Oxford: Elsevier. https://doi.org/10.1016/B978-0-08-097086-8.26087-X

Duckworth, A. L., Peterson, C., Matthews, M. D., \& Kelly, D. R. (2007). Grit: Perseverance and Passion for Long-Term Goals. Journal of Personality and Social Psychology, 92, 1087-1101. https://doi.org/10.1037/0022-3514.92.6.1087

Duckworth, A. L., Quinn, P. D., \& Seligman, M. E. P. (2009). Positive Predictors of Teacher Effectiveness. Journal of Positive Psychology, 4, 540-547. https://doi.org/10.1080/17439760903157232

Eskreis-Winkler, L., Shulman, E. P., Beal, S. A., \& Duckworth, A. L. (2014). The Grit Effect: Predicting Retention in the Military, the Workplace, School and Marriage. Frontiers in Psychology, 5, 36. https://doi.org/10.3389/fpsyg.2014.00036

Fite, R., Lindeman, M., Rogers, A., Voyles, E., \& Durik, A. (2017). Knowing Oneself and Long-Term Goal Pursuit: Relations among Self-Concept Clarity, Conscientiousness, and Grit. Personality and Individual Differences, 108, 191-194. https://doi.org/10.1016/j.paid.2016.12.008

Fleckenstein, J., Schmidt, F. T., \& Moeller, J. (2014). Who's Got Grit? Perseverance and Consistency of Interest in Pre-Service Teachers. A German Adaptation of the 12-Item Grit Scale. Psychologie in Erziehung und Unterricht, 61, 281-286. https://doi.org/10.2378/peu2014.art22d

Halliday, L., Walker, A., Vig, S., Hines, J., \& Brecknell, J. (2017). Grit and Burnout in UK Doctors: A Cross-Sectional Study across Specialties and Stages of Training. Postgraduate Medical Journal, 93, 389-394. https://doi.org/10.1136/postgradmedj-2015-133919

Hodge, B., Wright, B., \& Bennett, P. (2018). The Role of Grit in Determining Engagement and Academic Outcomes for University Students. Research in Higher Education, 59, 448-460. https://doi.org/10.1007/s11162-017-9474-y

Holdan, E. G., Lias, A. R., Locke, R. J., Elfen, H. H., \& Buzzelli, A. A. (2018). Success without Grit: An Exploratory Study of Individuals with Low Grit Scores and High Academic Performance. International Journal of Current Research, 10, 73250-73252.

Jachimowicz, J. M., Andreas, W., Bailey, E. R., \& Galinsky, A. D. (2018). Why Grit Requires Perseverance and Passion to Positively Predict Performance. Proceedings of the National Academy of Sciences, 115, Article ID: 201803561. https://doi.org/10.1073/pnas.1803561115

Jeong, J. Y., Seo, Y. S., Choi, J. H., Kim, S. H., Lee, M. S., Hong, S. H., Choi, J. S., \& Park, D. E. (2019). The Influence of GRIT on Turnover Intention of University Hospital Nurses: The Mediating Effect of Job Involvement. Journal Korean Society of Nursing Science, 49, 181-190. https://doi.org/10.4040/jkan.2019.49.2.181

Karaman, M. A., Vela, J. C., Aguilar, A. A., Saldana, K., \& Montenegro, M. C. (2018). Psychometric Properties of U.S.-Spanish Versions of the Grit and Resilience Scales with a Latinx Population. International Journal for the Advancement of Counselling, 41, 125-136. https://doi.org/10.1007/s10447-018-9350-2

Kazuji, N., Shiori, O., \& Toshihiko, A. (2015). Development of the Japanese Short Grit Scale (Grit-S). The Japanese Journal of Personality, 24, 167-169. https://doi.org/10.2132/personality.24.167

Kim, D., Choi, D., \& Vandenberghe, C. (2018). Goal-Focused Leadership, Leader Member Exchange, and Task Performance: The Moderating Effects of Goal Orientations and Emotional Exhaustion. Journal of Business and Psychology, 33, 645-660.

https://doi.org/10.1007/s10869-017-9516-7

Kim, Y. J., \& Lee, C. S. (2015). Effects of Grit on the Successful Aging of the Elderly in 
Korea. Indian Journal of Science and Technology, 8, 373-378.

https://doi.org/10.17485/ijst/2015/v8iS7/70421

Kleiman, E. M., Liu, R. T., \& Riskind, J. H. (2014). Integrating the Interpersonal Psychological Theory of Suicide into the Depression/Suicidal Ideation Relationship: A Short-Term Prospective Study. Behavior Therapy, 45, 212-221.

https://doi.org/10.1016/j.beth.2013.10.007

Latham, G. P., \& Pinder, C. C. (2005). Work Motivation Theory and Research at the Dawn of the Twenty-First Century. Annual Review of Psychology, 56, 485-516. https://doi.org/10.1146/annurev.psych.55.090902.142105

Lucas, G. M., Gratch, J., Cheng, L., \& Marsella, S. (2015). When the Going Gets Tough: Grit Predicts Costly Perseverance. Journal of Research in Personality, 59, 15-22. https://doi.org/10.1016/j.jrp.2015.08.004

Morton, J. M. (2014). Molding Conscientious, Hardworking, and Perseverant Students. Social Philosophy \& Policy, 31, 60-80. https://doi.org/10.1017/S0265052514000119

Muenks, K., Ji, S. Y., \& Wigfield, A. (2017). Associations between Grit, Motivation, and Achievement in High School Students. Motivation Science, 4, 158-176.

https://doi.org/10.1037/mot0000076

Pierce, J. R., \& Aguinis, H. (2013). The Effect in Management. Journal of Management, 39, 313-338. https://doi.org/10.1177/0149206311410060

Salles, A., Cohen, G. L., \& Mueller, C. M. (2014). The Relationship between Grit and Resident Well-Being. The American Journal of Surgery, 207, 251-254.

https://doi.org/10.1016/j.amjsurg.2013.09.006

Salles, A., Lin, D., Liebert, C., Esquivel, M., \& Mueller, C. (2016). Grit as a Predictor of Risk of Attrition in Surgical Residency. American Journal of Surgery, 213, 288-291. https://doi.org/10.1016/j.amjsurg.2016.10.012

Schimschal, S. E., \& Lomas, T. (2019). Gritty Leaders: The Impact of Grit on Positive Leadership Capacity. Psychological Reports, 122, 1449-1470. https://doi.org/10.1177/0033294118785547

Seguin, C. (2019). A Survey of Nurse Leaders to Explore the Relationship between Grit and Measures of Success and Well-Being. Journal of Nursing Administration, 49, 125-131. https://doi.org/10.1097/NNA.0000000000000725

Seo, K. S., Kim, M., \& Park, J. (2014). Effect of Resilience and Job Satisfaction on Organizational Commitment in Korean American Registered Nurses. Journal of Korean Academy of Nursing Administration, 20, 48-58.

https://doi.org/10.11111/jkana.2014.20.1.48

Sharkey, C. M., Bakula, D. M., Gamwell, K. L., Mullins, A. J., Chaney, J. M., \& Mullins, L. L. (2017). The Role of Grit in College Student Health Care Management Skills and Health-Related Quality of Life. Journal of Pediatric Psychology, 42, 73. https://doi.org/10.1093/jpepsy/jsx073

Sheldon, K. M., Jose, P. E., Kashdan, T. B., \& Jarden, A. (2015). Personality, Effective Goal-Striving, and Enhanced Well-Being: Comparing 10 Candidate Personality Strengths. Personality and Social Psychology Bulletin, 41, 575-585.

https://doi.org/10.1177/0146167215573211

Stoffel, J. M., \& Cain, J. (2018). Review of Grit and Resilience Literature within Health Professions Education. American Journal of Pharmaceutical Education, 82, 6150. https://doi.org/10.5688/ajpe6150

Suzuki, Y., Tamesue, D., Asahi, K., \& Ishikawa, Y. (2015). Grit and Work Engagement: A Cross-Sectional Study. PLOS ONE, 10, e0137501. 
https://doi.org/10.1371/journal.pone.0137501

Tyumeneva, Y., Kuzmina, J., \& Kardanova, E. (2014). IRT Analysis and Validation of the Grit Scale: A Russian Investigation. HSE Working Papers, National Research University Higher School of Economics. https://doi.org/10.2139/ssrn.2527859

Vainio, M. M., \& Daukantaite, D. (2015). Grit and Different Aspects of Well-Being: Direct and Indirect Relationships via Sense of Coherence and Authenticity. Journal of Happiness Studies, 17, 1-29. https://doi.org/10.1007/s10902-015-9688-7

Weisskirch, R. S. (2016). Grit, Self-Esteem, Learning Strategies and Attitudes and Estimated and Achieved Course Grades among College Students. Current Psychology, 37, 21-27. https://doi.org/10.1007/s12144-016-9485-4

Wright, T. A., \& Cropanzano, R. (2004). The Role of Psychological Well-Being in Job Performance: A Fresh Look at an Age-Old Quest. Organizational Dynamics, 33, 338-351. https://doi.org/10.1016/j.orgdyn.2004.09.002 